Brit. J. Psychiat. (1983), 143, 124-127

\title{
Quantitative Computed Tomography in Elderly Depressed Patients
}

\author{
ROBIN J. JACOBY, RAYMOND J. DOLAN, RAYMOND LEVY and ROBERT BALDY
}

\begin{abstract}
Summary: An index of brain tissue density was obtained from the CT scans of 37 elderly depressed patients by measuring Hounsfield Units (HU) in 12 predefined brain areas. Comparisons were made with 23 patients suffering from senile dementia and 36 healthy controls. As a group the depressed patients resembled the dements more than the controls, although analysis of variance indicated differences between all three groups, controls showing the highest $H U$ values and dements the lowest. In the depressed patients ventricular dilatation, which had already been shown to predict increased mortality, was also associated with lower HU levels. The significance of these and other findings is discussed.
\end{abstract}

The possible aetiological role of cerebral organic factors in depression in old age has been the subject of previous studies. Hemsi et al (1968) found alterations in the barbiturate threshold of elderly depressed patients, although the findings were not entirely confirmed in a later study (Davies et al, 1978). Hendrickson et al (1979) demonstrated a delay in the auditory evoked response (AER) in a similar group of patients. Furthermore, the delayed AER did not fall to the level of the healthy controls after treatment and recovery from depression. In our own computed tomographic (CT) study a sub-group of 9 (out of 41) late-onset elderly depressed patients with enlarged cerebral ventricles were identified, who were older and showed a more 'endogenous', clinical picture than the remainder (Jacoby and Levy, 1980b). In a follow-up study of this sample ventricular enlargement was found to predict an increased mortality at two years compared to both depressed patients without ventricular enlargement and to healthy controls (Jacoby et al, 1981).

These CT studies, in common with most of those from other authors, have assessed cerebral changes on the basis of enlargement of the CSF space, i.e. sulcal and ventricular dilatation. This approach is essentially similar to that used for pneumoencephalography (PEG) (Jacoby, 1981). However, the CT scanner allows examination of the brain substance, whereas PEG does not. The numerical data, or Hounsfield Units (HU), from the scanner's computer are a function of the radio-attenuation of the tissues through which the $x$-rays have passed, and therefore probably reflect brain tissue density. Some more recent studies, such as those by Naeser et al (1980) and Bondareff et al (1981) have found low HU in demented patients compared with normal controls. The present study aimed at using this new technique to seek further evidence of an aetiological role for cerebral organic change in depression in late life.

\section{Method}

(i) Subjects: These were drawn from three samples described previously (Jacoby et al, 1980; Jacoby and Levy, 1980a and b), comprising 41 elderly patients with affective disorder, 40 patients with senile dementia, and a control group of 50 healthy volunteers. The subjects for this study were those whose original CT scans were of sufficient quality to enable the quantitative analysis to be made by virtue of minimal or absent movement artefact. Thirty-seven patients with affective disorder, 23 with dementia and 36 controls were available for study.

(ii) CT Scans: In the original study scans were 'blindly' rated by a neuroradiologist, who made a global assessment of ventricular size and gave an index of sulcal dilatation, the cortical atrophy score (CAS), for five cortical areas-frontal, temporal, insulae, parietal, occipital. The ventricle-brain ratio (VBR) was measured by planimetry.

In the present study $C T$ scans, which had been stored on magnetic tape, were displayed on the television monitor of an Independent Viewing Centre (IVC). A tracker ball was used to define an area of 129 pixels in 12 predetermined brain regions (six left and six right) on the various slices in which they were optimally displayed. The regions were anterior frontal, 
posterior frontal, temporal, thalamus, parietal and occipital. Mean Hounsfield Units (HU) were measured for each 129 pixel area.

(iii) Reliability: All HU measurements used in the statistical analysis were made by one author (RD). In order to test the inter-rater reliability of the method 78 measurements were made on a separate occasion by another author (RJ), slices and areas being chosen randomly from all three groups of subjects. Reliability was assessed by subtracting each measurement of one observer from the equivalent one of the other observer and calculating the mean (SD) of the differences. The correlation coefficient between the two sets of measurements was also calculated.

(iv) Statistical analysis: Mean HU were compared for individual brain areas by separate t-tests. Univariate analysis of variance (anova) was employed to test for group differences where density (HU) was the dependent variable, and side (left/right), brain area, and diagnosis were the independent variables. Correlation coefficients of HU with CAS, VBR and age were also calculated. $t$-Tests were used to compare HU in depressed patients who had received ECT in the past with those who had not received ECT.

\section{Results}

(i) Reliability: The means (SD) of 78 measurements by the two observers were 36.57 (4.07) and 36.07 (4.28) respectively. The mean of the differences was 0.47 (1.40). The correlation (Pearson's r) between the two sets of measurements was 0.945 .

(ii) Diagnostic differences: The distribution of $\mathrm{HU}$ within each diagnostic group was normal (gaussian). For individual brain areas there was no significant difference on separate $t$-tests between the demented and the depressed patients. The significant differences between the patients and controls are shown in Table I.

In the analysis of variance the null hypothesis that density (HU) was the same in the three diagnostic groups was not upheld $(F=11.45, P<0.001)$; nor was

$$
\text { TABLE I }
$$

Differences between patients and controls mean (SD) Hounsfield Units

\begin{tabular}{llll}
\hline & \multicolumn{1}{c}{ Dementia } & \multicolumn{1}{c}{ Affective } & \multicolumn{1}{c}{ Control } \\
\hline L Temporal & $34.9(4.5)^{*}$ & $34.8(4.5)^{* *}$ & $38.0(4.5)$ \\
R Temporal & $35.1(4.5)^{*}$ & $35.9(4.9)$ & $37.4(4.1)$ \\
L Thalamus & $32.1(2.6)^{* *}$ & $33.4(2.8)$ & $34.5(3.1)$ \\
R Thalamus & $32.1(2.6)^{* * *}$ & $32.7(2.8)^{* *}$ & $34.8(2.9)$ \\
L Parietal & $32.9(3.0)^{\prime \prime}$ & $33.6(4.4)$ & $34.5(3.7)$ \\
R Parietal & $32.6(3.9)^{*}$ & $34.3(4.4)$ & $34.3(3.1)$ \\
\hline
\end{tabular}

2-tail significant difference from control (t-test):

* $P<0.05 ; * * P<0.005 ;{ }^{* * *} P<0.001$

1-tail significant difference from control (t-test):

" $\mathrm{P}<0.05$ it upheld with respect to variation between brain areas $(F=20.56, P<0.001)$. However there were no significant left/right differences.

(iii) Within group differences: In every brain area the nine depressed patients found by the neuroradiologist to have enlarged ventricles showed lower mean HU than the depressed patients with normal ventricles. The data are shown in Table II. Analysing the depressed group separately these differences were significant when ventricular enlargement (the radiologist's rating) was introduced into the anova as an independent variable $(F=18.86, P<0.001)$. By contrast, the controls with enlarged ventricles showed higher mean $\mathrm{HU}$ than controls with normal ventricles $(F=10.99, P<0.001)$. Separation of samples on the basis of the radiologists' subjective rating of ventricular size was validated by comparing the sub-groups in respect of VBR. In both the affective and control groups those subjects considered by the radiologist to have global ventricular enlargement showed a greater

TABLE II

Mean (SD) Hounsfield Units in depressed patients with and without enlarged ventricles

\begin{tabular}{|c|c|c|}
\hline Brain area & Ventricles enlarged & Ventricles normal \\
\hline $\begin{array}{l}\text { L temporal } \\
R \text { temporal } \\
\text { L thalamus } \\
R \text { thalamus } \\
\text { L anterior frontal } \\
R \text { anterior frontal } \\
\text { L posterior frontal } \\
R \text { posterior frontal } \\
\text { L parietal } \\
R \text { parietal } \\
\text { L occipital } \\
\text { R occipital }\end{array}$ & $\begin{array}{l}33.3(2.6) \\
34.9(3.1) \\
32.2(2.7) \\
31.5(2.4) \\
34.7(5.5) \\
32.1(5.7) \\
32.0(6.4) \\
31.1(7.7) \\
31.6(2.1) \\
32.9(2.9) \\
30.8(5.5) \\
32.4(4.2)\end{array}$ & $\begin{array}{l}35.3(4.9) \\
36.2(5.4) \\
33.8(2.9) \\
33.1(2.9) \\
36.1(5.3) \\
35.8(5.5) \\
35.9(4.4) \\
36.0(4.7) \\
34.2(4.9) \\
34.8(4.9) \\
33.6(4.7) \\
33.3(5.6)\end{array}$ \\
\hline Mean of all areas & $\begin{array}{c}32.5(4.6) \\
F=18.86, P<0.001\end{array}$ & $34.8(4.7)$ \\
\hline
\end{tabular}

mean (SD) VBR than subjects with normal ventricles: 18.7 (2.8) cf 14.1 (4.0), $P<0.005$-affectives; 19.1 (3.6) cf 13.2 (3.2), $\mathrm{P}<0.001-$ controls. There were too few demented patients (5) with normal ventricles in whom HU could also be measured for statistical analysis.

(iv) Relationship of $\mathrm{HU}$ to other variables. There was no significant correlation between $\mathrm{HU}$ and age in any of the diagnostic groups. A previous history of having received ECT was not related to $\mathrm{HU}$. In the demented patients and controls there was no correlation between HU and CAS or VBR. The significant correlations in the affective group are shown in Table III. 
TABLE III Correlation of $H U$ with $C A S$ and $V B R$ affective group only

\begin{tabular}{cc}
\hline Brain area & Pearson's r \\
\hline CAS with L temporal & $-0.395^{* \prime \prime}$ \\
L posterior frontal & $-0.403^{* \prime}$ \\
R posterior frontal & $-0.474^{* *}$ \\
L parietal & $-0.445^{* *}$ \\
R parietal & $-0.389^{* \prime \prime}$ \\
\hline VBR with L posterior frontal & $-0.338^{* \prime \prime}$ \\
R posterior frontal & $-0.329^{* \prime \prime}$
\end{tabular}

* $\mathrm{P}<0.05 ; \quad$ * $\mathrm{P}<0.01 ; \quad$ "NS when patients with enlarged ventricles omitted.

All the significance levels in Table III were reduced when the subgroup with enlarged ventricles was omitted from the analysis.

\section{Discussion}

(i) Validity and reliability: There are some theoretical sources of error in the technique of measuring $\mathrm{HU}$. Low readings may be made in some brain areas due to partial volume artefact, a detailed explanation of which was given by Jacoby et al (1980). In summary, because each tomogram is the two-dimensional representation of a three-dimensional structure, some pixels at brainventricle interfaces show an attenuation density which is partly due to brain tissue and partly due to CSF. A further possible source of error could be machine drift, which is the alteration over time in output (HU) from a single scanner. This alteration is usually slight, and the calibration of scanners is regularly adjusted against phantoms of known radio-attenuation.

The low mean of the differences and the high correlation between the two sets of observations in this study suggest that this is a reliable method of measuring HU. One caveat is that the data which some of us reported elsewhere (Bondareff et al 1981) using some of the same subjects and the same IVC showed higher absolute HU, although the relative diffferences between dements and controls (depressives were not measured) were the same. Bondareff's paper did not entail a reliability study, but possible explanations for the discrepancy may be technical rather than observer error. For instance there were some changes to the computer programme between the two studies, notably to exclude HU greater than 70 which were considered likely to be artefact, eg calcification. Also, Bondareff defined a larger area than the 129 pixels covered in this study; the purpose of the change being to pick out more discrete areas of the brain. However, it can be argued that the discrepancy between the two studies is not serious since relative differences were preserved. (ii) Diagnostic differences: One of the most striking findings in this study was that mean HU for most brain areas in the depressed patients more closely resembled the dements than the controls. This is of particular interest as the CT indices in our original study (VBR, CAS and radiologist's rating of ventricular size) suggested that the depressed group was more like the controls than the dements. However, although the depressed patients were closer to the demented in respect of mean $\mathrm{HU}$, analysis of variance indicated that they constituted a significant intermediate group between dements and controls. This finding reflects those of Hendrickson et al (1979) for the delay in the auditory evoked response.

(iii) Within group differences: It is also of interest that it was the sub-group of nine patients with enlarged ventricles, identified in the first study (Jacoby and Levy, 1980b), and found to have suffered an excess mortality in the second (Jacoby et al, 1981), which accounted for a significant amount of the variance in HU between the whole affective group and the other two groups in the study. This association between ventricular enlargement and low $\mathrm{HU}$ is the more interesting since it was confined to the depressed patients and not found in controls, suggesting that ventricular enlargement does not necessarily imply low brain density. It should be emphasised here that follow-up of the depressed patients did not indicate that they were a sub-group of undiagnosed cases of dementia (Jacoby et al, 1981). It seems possible, therefore, that ventricular enlargement in these patients reflected a different pathological process from that of senile dementia of the Alzheimer type. Whether or not it is a non-specific loss of neurones and glial cells, or whether it is more specific remains to be determined by an investigation of autopsy material which was not possible with these patients. Some evidence that the process may be a relatively diffuse one is suggested by the several significant correlations between $\mathrm{HU}$ and CAS, the index of sulcal dilatation, in Table III, where 5 out of 12 significant correlations in the depressed group alone is unlikely to have been a chance finding.

If low HU in the depressed group represents a nonspecific ageing of the CNS (Post, 1968), it may not necessarily be a chronological phenomenon, since there was no correlation between $\mathrm{HU}$ and chronological age in any of the three diagnostic groups.

(iv) ECT. An earlier study by some of us on this series of depressed patients showed an association between sulcal dilatation in the frontal lobes and a history of having had ECT (Calloway et al, 1981). This finding was not supported by the present study, although it cannot be assumed that the two processes resulting in sulcal dilatation and lowered density 
respectively are necessarily the same. Indeed, some evidence has emerged here that they are not so, since the controls with enlarged ventricles did not show low HU levels. However, our earlier findings with regard to ECT were considered tentative only, indicating the need for a more systematic investigation.

\section{Acknowledgements}

Our sincere thanks are due to Mr G. B. Newman of the Computer Services Department at the Middlesex Hospital for statistical advice. The IVC was provided under an MRC programme grant to Professor W. A. Lishman, who kindly allowed us this facility. Robert Baldy is in receipt of an MRC grant. This research forms part of a series of investigations supported by a grant to Dr R. Levy from the Board of Governors of the Bethlem Royal Hospital and the Maudsley Hospital.

\section{References}

BondarefF, W., Baldy, R., \& LeVY, R. (1981) Quantitative computed tomography in senile dementia. Archives of General Psychiatry, 38, 1365-8.

Calloway, S. P., Dolan, R. J., Jacoby, R. J. \& LeVy, R. (1981) ECT and cerebral atrophy: a computed tomographic study. Acta Psychiatrica Scandinavica, 64, 442-5.

Davies, G., Hammion, S., Hendrickson, D. E., Levy, R. \& Post, F. (1978) Psychological test performance and sedation thresholds of elderly dements, depressives and depressives with incipient brain damage. Psychological Medicine, 8, 103-9.
Hemsi, L. K., Whitehead, A. \& Post, F. (1968) Cognitive function and cerebral arousal in elderly depressives and dements. Journal of Psychosomatic Research, 12, 14556.

Hendrickson, E., Levy, R. \& Post, F. (1979) Averaged evoked responses in relation to cognitive and affective state of elderly psychiatric patients. British Journal of Psychiatry, 134, 494-501.

JACOBY, R. J. (1981) Dementia, depression and the CT scan. Psychological Medicine, 11, 673-6.

— LeVY, R. \& Dawson, J. M. (1980) Computed tomography in the elderly: 1 The normal population. British Journal of Psychiatry, 136, 249-55.

(1980a) Computed tomography in the elderly: 2 Senile dementia: diagnosis and functional impairment. British Journal of Psychiatry, 136, 256-69.

- (1980b) Computed tomography in the elderly: 3 Affective disorder. British Journal of Psychiatry, 136, 270-5.

- \& BiRD, J. M. (1981) Computed tomography and the outcome of affective disorder: a follow-up study of elderly patients. British Journal of Psychiatry, 139, 288-92.

Naeser, M. A., Gebhardt, C. \& LeVine, H. L. (1980) Decreased computerized tomography numbers in patients with presenile dementia: detection in patients with otherwise normal scans. Archives of Neurology, 37, 401-9.

Post, F. (1968) The factor of ageing in affective illness. In Recent Developments in Affective Disorders (eds A. Coppen and A. Walk). Ashford, Kent: Headley Brothers.

Robin J. Jacoby, D.M., M.R.C.P., M.R.C.Psych., Senior Lecturer and Consultant Psychiatrist, The Middlesex Hospital, Mortimer Street, London W1N 8AA

* Present address: The Maudsley Hospital, Denmark Hill, London, SE5 8AZ

Raymond J. Dolan, M.B., B.Ch., M.R.C.Psych., Wellcome Research Fellow and Honorary Senior Registrar, The Royal Free Hospital, Pond Street, London NW3 2QG

Raymond Levy, PhD., F.R.C.P. (Ed)., F.R.C.Poych., Consultant Psychiatrist, The Maudsley Hospital

Robert Baldy, L\& L., Research Worker, The Maudsley Hospital

*Correspondence. 